Link operations for slowing the spread of disease in complex networks

This article has been downloaded from IOPscience. Please scroll down to see the full text article.

2011 EPL 9518005

(http://iopscience.iop.org/0295-5075/95/1/18005)

View the table of contents for this issue, or go to the journal homepage for more

Download details:

IP Address: 150.203.162.16

The article was downloaded on 03/05/2012 at 05:57

Please note that terms and conditions apply. 


\title{
Link operations for slowing the spread of disease in complex networks
}

\author{
A. N. Bishop ${ }^{1(a)}$ and I. $\operatorname{ShAmes}^{2(b)}$ \\ ${ }^{1}$ NICTA, Canberra Research Laboratory and the Australian National University (ANU) - Canberra, Australia \\ ${ }^{2}$ ACCESS Linnaeus Centre, Royal Institute of Technology - Stockholm, Sweden, EU
}

received 27 January 2011; accepted in final form 20 May 2011

published online 21 June 2011

PACS 89.75. Hc - Networks and genealogical trees

PACS 88.80.Cd - Grid-connected distributed energy resources

PACS 89.65.Ef - Social organizations; anthropology

\begin{abstract}
A variety of social, biological and communication networks can be modelled using graph theoretical tools. Similar graphical tools can be used to model the topology by which disease, errors, and/or other undesired phenomenon etc. is spread and propagated through such networks. Certain network operations are proposed in this work that can be used to slow the spread of diseases in complex network topologies. The approach considered in this work differs from existing techniques in that it is based on optimally removing (or immunizing) individual links in the network as opposed to individual nodes. A systematic algorithm is outlined to achieve this edgewise immunization via a relaxed convex optimization protocol.
\end{abstract}

Copyright (c) EPLA, 2011

Introduction. - A variety of complex interactions between individual entities can be accurately modelled on top of a network consisting of nodes that represent the entities and links which represent the interactions between entities $[1,2]$. Social and biological interactions between living entities can be modelled as links between nodes in a graph [3-5]. Communication networks have long been modelled using graph theoretic models in both the social/biological and electrical engineering domains [3,5]. Other, complex infrastructure, networks such as the power, water and cyber networks are also modelled using graphs [2].

Apart from modelling the specific interactions between individual entities, e.g. the communication between computers on a cyber network, the same graph theoretic tools can be used to model the topology by which disease, errors, and/or other undesired phenomenon etc. is spread and propagated through such networks $[5,6]$. For example, a graph used to model the cyber network can also highlight the paths along which certain computer viruses can spread. The actual spread of the disease ${ }^{1}$ between entities (and thus on the network proper) is

\footnotetext{
(a) E-mail: adrian.bishop@anu.edu.au

(b) E-mail: imansh@kth.se.

${ }^{1}$ The idea of a disease is quite general and this work is applicable to the spread of worms/viruses in networked computer systems, the spread of cascading failures in networked electrical systems (such as
}

typically modelled probabilistically using a Markov chain or dynamical mean-field equations.

The dynamics of the disease propagation, e.g. steadystate characteristics etc., have been explicitly related to certain graph theoretical properties of the network itself, e.g. the eigenvalues of the adjacency matrix. A wide range of results have been expressed in the literature, e.g., see [7-12], where different random and deterministic graph topologies, e.g. small-world, Erdös-Rényi, geometric etc., and different propagation characteristics, e.g. concerning specific viruses or infection/susceptibility models etc., have been considered. We refer to the references for details.

Given the natural connection between the dynamics of the disease propagation and the topology of the underlying network it follows that one may seek to apply certain topological operations to the network with the aim of slowing/reducing the spread of disease or increasing the eradication of the disease from the network. This is exactly the topic of this work where we consider certain network edge-based operations that can be used to slow the spread of diseases in complex network topologies. Conversely, such operations increase the rate in which a disease may die out in similarly complex networked environments.

the smart grid) or the spread of epidemics in biological networks. We will use the word disease as a catch all term. 
Other work that examines methods to reduce the effect of a disease in a network is explored in [13-21] where it is typical to immunize certain individual nodes, e.g. the nodes with the highest degree or a certain fraction of the nodes etc. We refer to these references also for comparisons with the effect of random node immunization. From the networks perspective, immunizing individual nodes is equivalent to removing each edge incident on that node (at least from the point of view concerning the spread of disease). This is true regardless of how the individual nodes are chosen.

The approach considered in this work differs from these preceding immunization strategies in a fundamental way. The strategy considered in this work is based on optimally removing (or immunizing) individual links in the network. Thus, individual nodes are not necessarily immunized in the traditional sense but rather we actively control the interactions and the disease's path of movement. At a basic level, we consider the immunization of individual links as opposed to the immunization of individual nodes. Our goal is to introduce edge-based operations that can be used to slow the spread of, or eliminate entirely, diseases in complex network topologies. We provide a systematic algorithm to achieve this immunization via a relaxed convex optimization protocol.

To motivate the idea of edgewise immunization consider a social network of organisms susceptible to a disease that is spread via links represented using a graph theoretical model. Suppose also that the links represent the social interactions between organisms and it is this social interaction that facilitates the ability of a disease to spread. Then, our claim is that if we simply restrict specific social interactions between strategic pairs of organisms, then we could achieve a kind of edgewise immunization of the network; e.g. we could slow the spread of the disease significantly or conversely increase the rate in which the disease becomes extinct. This scheme does not require one to completely isolate individual organisms from their neighbours in the network. Indeed, one can then contrast the difference between the total quarantine of individual organisms in the network (i.e. node-based immunization) with strategic pairwise interaction restriction (i.e. edgewise immunization).

Another example of where one may wish to consider socalled edgewise immunization is during the network design stage for critical networked infrastructure; e.g. power control or transportation control networks etc. Often, these networks are fixed, involved distributed processing, and are required to be connected. Moreover, we would increasingly like such a network to be resilient to the so-called zero-day attacks and vulnerabilities. A zero-day attack refers to a virus or fault that attacks, often maliciously, a network (most typically a computer network or more recently an industrial control and process network) but which is otherwise unknown to the system security analysts and designers etc. By designing the network topology using the tools developed in this work one can achieve a sort of edgewise immune network topology that may yield a greater network resilience to the so-called zero-day attacks while also aiding in the recovery from such attacks; e.g. by ensuring any zero-day disease entering the network will propagate slower and/or die out faster once a counter-action is established.

The key question considered is then: What edges in the network should be removed in order to best facilitate the extinction of the disease from the network? This idea outlines a novel direction for immunization in complex networks and it is envisioned that such a scheme can find application in a variety of settings including the power, water and cyber networks.

A model for the spread of disease in complex network. - We suppose there are $n$ nodes whose connections are represented by a network (or graph) and are completely characterized by the entries $a_{i j}$ of an associated adjacency matrix $\mathbf{A}$. The network is formally described by the graph $\mathcal{G}=\{\mathcal{V}, \mathcal{E}\}$, where $\mathcal{V}$ is the set of nodes and the set $\mathcal{E}$ is the set of connections (or edges). The neighbours of node $i$ are denoted by $\mathcal{N}_{i}=\{j \in \mathcal{V} \mid(i, j) \in \mathcal{E}\}$.

Let the time $t \in \mathbb{N}$. The infected nodes attempt (either maliciously or by circumstance) to infect their susceptible neighbours with probability $\beta$. An infected node has a probability of $\mu$ of recovering from the infection and returning to a susceptible state. Let $p_{i}(t)$ denote the probability that node $i$ is infected at time $t$ and let $\mathbf{p}(t)=\left[p_{1}(t) \ldots p_{n}(t)\right]^{\top}$. One can then derive an equation of the form

$$
\mathbf{p}(t+1)=f(\mathbf{p}(t), \beta, \mu),
$$

where $f(\mathbf{p}(t), \beta, \mu)$ is a function detailing the evolution of the disease through the network. For example, $f(\mathbf{p}(t), \beta, \mu)$ should account for the possibility that, e.g., node $i$ is susceptible and infected by a neighbour, that node $i$ is infected and does not recover at time $t$ and that an infected node recovers and is subsequently re-infected by a neighbour within the same discrete time window.

Note, we point to [7-12] for details concerning various systems of the form (1) used to model the spread of disease in a network.

The work in [7-12] is noteworthy since it relates the behaviour of systems of the form (1) to the network topology defined by, e.g., A. That is, these references relate the behaviour of the disease in the network, e.g. its propagation rate or steady-state behaviour, to the network topology.

The following is an important result in networked disease propagation and has been mentioned by a number of authors studying different, but closely related, systems of the form (1).

Theorem 1 ([8,11]). If $\frac{\beta}{\mu}<\frac{1}{\left|\lambda_{\max }(\mathbf{A})\right|}$ then $\mathbf{p}(t) \rightarrow \mathbf{0}$ as $t \rightarrow \infty$ with an exponential rate.

This result has continuous time analogous and a number of comments on an inequality of the form $\frac{\beta}{\mu}<\frac{1}{\left|\lambda_{\max }(\mathbf{A})\right|}$ 
have been stated in various settings; e.g. see [7-12] and the references therein. For brevity, we highlight $[8,11]$ which contain mathematically rigorous results concerning the evolution of a system of the form (1). In [8] the authors examine the rate at which a disease dies out and is eliminated from the network. The authors show that if $\frac{\beta}{\mu}<\frac{1}{\left|\lambda_{\max }(\mathbf{A})\right|}$ does not hold then the disease may persist in the network for a very long time. In [11] the authors provide a detailed analysis for a similar problem by considering a mean-field approximation of an exact $2^{n}$ state Markov process that captures all the possible combinations of the network state. Among other results, the authors find that $\frac{\beta}{\mu}<\frac{1}{\left|\lambda_{\max }(\mathbf{A})\right|}$ ensures the disease is removed from the network. This formulation highlights that, because $2^{n}$ will be quite large in many cases, the features of an infinite-state Markov process rapidly come into play. This also leads the authors to conclude that if $\frac{\beta}{\mu}<\frac{1}{\left|\lambda_{\max }(\mathbf{A})\right|}$ does not hold then the disease may persist in the network for a very long time.

The practical consequence of this theorem, as it pertains to this work, is that one can deduce the existence of some critical probability $\beta_{c} \approx \mu /\left|\lambda_{\max }(\mathbf{A})\right|$ below which the disease dies out quickly and above which the disease may, and quite often does, persist in the network for a long time.

A strategy for selective immunization. - The main contribution of this letter is the introduction of a mechanism by which one can systematically adjust the network topology in order to increase the speed in which the disease is removed from the network. In the previous section it was noted that a smaller maximum eigenvalue for the adjacency matrix of the underlying network graph reduces the critical infection probability and increases the rate at which the disease dies out. Heuristically, this is not surprising. In this section we propose a systematic method to change the structure of the network through removing links (or edges in the graph) to minimize this eigenvalue.

The basic solution. In this subsection we consider the problem of determining which edges to remove from the network in order to reduce the spectrum of the adjacency matrix. We constrain the number of edges that can be removed to $m_{e}$.

First note that the adjacency matrix of a graph $\mathcal{G}, \mathbf{A}$ can be written as

$$
\mathbf{A}=\sum_{i=1}^{|\mathcal{E}|} \mathbf{A}_{i}
$$

where $\mathbf{A}_{i}$ is a matrix with all zero entries except for the $i j$-th and $j i$-th entries which are equal to 1 . The problem of minimizing $\lambda_{\max }$ can the formulated as

$$
\begin{array}{ll}
\operatorname{argmin}_{\mathbf{s}} \quad \lambda_{\max }\left(\sum_{i=1}^{|\mathcal{E}|} s_{i} \mathbf{A}_{i}\right), \\
\text { subject to } \mathbf{1}^{\top} \mathbf{s}=|\mathcal{E}|-m_{e}, \\
s_{i} \in\{0,1\}, \quad i=1, \cdots,|\mathcal{E}|,
\end{array}
$$

where $\lambda_{\max }(\cdot)$ is the largest eigenvalue of its argument.

Because the constraint $s_{i} \in\{0,1\}$ is Boolean, (2) is not easily solvable using standard convex optimization techniques. However, one can relax this problem and obtain a convex optimization problem that can be solved using standard tools ${ }^{2}$. We have the following relaxed version of (2):

$$
\begin{array}{ll}
\operatorname{argmin}_{\mathbf{s}} & \lambda_{\max }\left(\sum_{i=1}^{|\mathcal{E}|} s_{i} \mathbf{A}_{i}\right), \\
\text { subject to } & \mathbf{1}^{\top} \mathbf{s}=|\mathcal{E}|-m_{e} \\
& s_{i} \in[0,1], \quad i=1, \cdots,|\mathcal{E}|
\end{array}
$$

Now observe that the cost function in (3) is a convex function in $\mathbf{s} \in[0,1]^{|\mathcal{E}|}$ and the constraint is linear. To see this note firstly that $\sum_{i=1}^{|\mathcal{E}|} s_{i} \mathbf{A}_{i}$ is symmetric and, when viewed as a function of $\mathbf{s} \in[0,1]^{|\mathcal{E}|}$, has a convex domain. Moreover,

$$
\begin{aligned}
& \lambda_{\max }\left(\sum_{i=1}^{|\mathcal{E}|} s_{i} \mathbf{A}_{i}\right)= \\
& \sup _{\mathbf{x} \in \mathbb{R}^{|\mathcal{V}|}}\left\{\mathbf{x}^{\top}\left(\sum_{i=1}^{p} s_{i} \mathbf{A}_{i}\right) \mathbf{x}:\|\mathbf{x}\|=1\right\}:[0,1]^{|\mathcal{V}| \times|\mathcal{V}|} \rightarrow \mathbb{R}
\end{aligned}
$$

shows that $\lambda_{\max }\left(\sum_{i=1}^{|\mathcal{E}|} s_{i} \mathbf{A}_{i}\right)$ can be viewed as a pointwise supremum over an infinite set of linear functionals of $\sum_{i=1}^{|\mathcal{E}|} s_{i} \mathbf{A}_{i}$ indexed by $\mathbf{x} \in \mathbb{R}^{|\mathcal{V}|}$. Now it follows, e.g. from [22], that the pointwise supremum over a set of convex functionals preserves convexity. Thus, (3) is a convex problem as claimed.

The main difficulty with (3) is that it is nondifferentiable in the $s_{i}$. However, one can form a semi-definite programming (SDP) problem

$$
\begin{array}{ll}
\operatorname{argmin}_{\mathbf{s} \in[0,1]|\mathcal{E}|, t \in \mathbb{R}} t, & \\
\text { subject to } & t \mathbf{I}-\left(\sum_{i=1}^{|\mathcal{E}|} s_{i} \mathbf{A}_{i}\right) \geqslant \mathbf{0}, \\
& \mathbf{1}^{\top} \mathbf{s}=|\mathcal{E}|-m_{e},
\end{array}
$$

where we have used the property that $\lambda_{\max }\left(\sum_{i=1}^{|\mathcal{E}|} s_{i} \mathbf{A}_{i}\right) \leqslant$ $t$ if and only if $\sum_{i=1}^{|\mathcal{E}|} s_{i} \mathbf{A}_{i} \leqslant t \mathbf{I}$. The above minimization problem can thus be solved easily using standard SDP

\footnotetext{
${ }^{2}$ The original, exact, optimization problem is a combinatorial problem. The complexity of solving the original problem is on the order of $\left(\begin{array}{c}|\mathcal{E}| \\ m_{e}\end{array}\right)$ times the complexity of calculating the maximum eigenvalue of an $n$ by $n$ matrix. Except for the most trivial cases, the exact problem cannot be solved in any reasonable time frame. However, there are efficient tractable algorithm to solve the relaxed problem. Of course, one would expect that the solution to the (2) relaxed problem is generally not the same as the solution to the exact problem. The deviation of the relaxed solution from the exact solution and its relationship to the relative computational complexity is beyond the scope of this article and is a topic for future work.
} 
tools; we refer to [22] for details on SDP and its application to optimization of certain matrix properties including eigenvalues.

Assume now that $\mathbf{s}^{*}$ is the solution to this problem; it is not necessarily a solution to the original problem, since $s_{i}^{*}$ can take a non-integer value in $[0,1]$. However, one can say that the value of the objective function for $\mathbf{s}^{*}$ is an upper bound for the value of the objective function at a solution of the original problem because the feasible set for the relaxed problem contains the solution to the original problem. Call this upper bound $u_{s}$. To generate a possibly suboptimal solution to the non-relaxed problem we can proceed as follows. Let

$$
s_{i_{1}}^{*} \geqslant s_{i_{1}}^{*} \geqslant \ldots \geqslant s_{i_{|\mathcal{E}|}}^{*}
$$

denote the elements of $\mathbf{s}^{*}$ rearranged in descending order. Now compose the $|\mathcal{E}|$-vector $\hat{\mathbf{s}}$ with entries $\hat{s}_{i} \in\{0,1\}$ such that $\hat{s}_{i_{k}}=1$ for $k \in\left\{1, \cdots,|\mathcal{E}|-m_{e}\right\}$ and $\hat{s}_{i_{k}}=0$ for $k \in\left\{|\mathcal{E}|-m_{e}+1, \cdots,|\mathcal{E}|\right\}$. The entries of $\hat{\mathbf{s}}$ with indices corresponding to the $|\mathcal{E}|-m_{e}$ largest elements of $\mathbf{s}^{*}$ are assigned to be unity and the rest to be zero. The associated objective value with $\hat{\mathbf{s}}$ given by $\ell_{\mathbf{s}}$ is a lower bound for the optimal objective function. We define a gap between the upper bound and the lower bound as $\delta_{s}=u_{s}-\ell_{s}$.

One can then use $\delta_{s}$ to estimate the quality of the solution $\hat{\mathbf{s}}$ in solving the original problem (2). If $\delta_{s}$ is not negligible then a local optimization method can be used if desired to seek a better solution. We then remove, or immunize, those $m_{e}$ edges associated with the $m_{e}$ smallest $s_{i}$.

The algorithm proposed is a systematic method to minimize the maximum eigenvalue of the adjacency matrix by strategically removing links using a relaxed convex optimization protocol. The idea is that by minimizing the maximum eigenvalue of the adjacency matrix we simultaneously increase the critical probability $\beta_{c} \approx \mu /\left|\lambda_{1}\right|$. A disease is likely to die out faster when the infection probability between pairs of nodes $\beta$ is below $\beta_{c}$ and thus maximizing $\beta_{c}$ is desirable.

A generalized solution. Suppose that some desired critical value is given $\beta_{c}^{*}$. We constrain the number of edges that can be removed to $m_{e}$. Moreover, suppose some set $\mathcal{E}_{c} \subseteq \mathcal{E}$ of edges is given and each edge in $\mathcal{E}_{c}$ is an edge that must remain in the network $\mathcal{G}$ following the edge-removal procedure. Then $|\mathcal{E}|-\left|\mathcal{E}_{c}\right|>m_{e}$ is a necessary condition for feasibility. Finally, suppose one may wish the resulting network to be connected; we define a switch $\mathcal{C}=1$ if the network must remain connected with $\mathcal{C}=0$, otherwise. Note if $\mathbf{A}(\mathbf{s})=\sum_{i=1}^{|\mathcal{E}|} s_{i} \mathbf{A}_{i}$ with $s_{i} \in\{0,1\}$ as before then the Laplacian matrix is $\mathbf{L}(\mathbf{s})=\mathbf{D}(\mathbf{s})-\mathbf{A}(\mathbf{s})$, where $\mathbf{D}(\mathbf{s})$ is a weighted graph degree matrix dependent on $\mathbf{s}$. The eigenvalues of $\mathbf{L}(\mathbf{s})$ are arranged as $0=\lambda_{0} \leqslant \lambda_{1} \leqslant \ldots \leqslant \lambda_{|\mathcal{V}|}$ where the smallest eigenvalue is always equal to zero and the second smallest eigenvalue $\lambda_{1}(\mathbf{s})>0$ if and only if the network is connected; e.g. see [23] for further details. Note $\lambda_{1}(\mathbf{s})$ is a concave function in $\mathbf{s}$, e.g. see [24].
Consider now the following optimization problem:

$$
\begin{array}{ll}
\operatorname{argmin} & \lambda_{\max }\left(\sum_{i=1}^{|\mathcal{E}|} s_{i} \mathbf{A}_{i}\right) \\
\text { subject to } & \mathbf{1}^{\top} \mathbf{s}=|\mathcal{E}|-m_{e}, \\
& \lambda_{\max }\left(\sum_{i=1}^{|\mathcal{E}|} s_{i} \mathbf{A}_{i}\right)>\beta_{c}^{*}, \\
& \lambda_{1}(\mathbf{s})>0, \text { if } \mathcal{C}=1, \\
& s_{j} \in\{0,1\}, j=1, \cdots,|\mathcal{E}|-\left|\mathcal{E}_{c}\right|, \\
& s_{j}=1, j>|\mathcal{E}|-\left|\mathcal{E}_{c}\right|,
\end{array}
$$

where the optimization problem is over the $s_{j} \in\{0,1\}$ with $j$ indexing those edges in $\mathcal{E} \backslash \mathcal{E}_{c}$ only. The weight $s_{k}=1$ is essentially removed from the problem for those edges indexed by $k$ in $\mathcal{E}_{c}$.

If a solution to this problem exists, then it can be approximated via the same kind of relaxation, convex SDP formulation and subsequent edge removal procedure described above. In general, a solution, if it exists, removes those edges in $\mathcal{E} \backslash \mathcal{E}_{c}$ such that $\beta_{c} \approx \mu /\left|\lambda_{1}\right|$ is driven above a certain desired critical value $\beta_{c}^{*}$ while the network is forced to be connected. For example, suppose that both $\beta$ and $\mu$ are known in advance, then we may want $\beta_{c}^{*}=$ $\mu / \beta \rightarrow \beta_{c}>\beta$.

Some of the constraints may be removed dependent on the scenario; e.g. one may not care which edges are removed so that $\mathcal{E}_{c}=\emptyset$. Note this problem may have no solution in general; e.g. one can easily imagine if $\beta_{c}^{*}$ is large enough, $\mathcal{E}_{c} \approx \mathcal{E}$ but with $\left|\mathcal{E}_{c}\right|<|\mathcal{E}|$ and one wishes the network to be connected then a solution is unlikely to exist.

Simulation results and comparison. - In this section we test the performance of the method introduced in this paper for minimizing the maximum eigenvalue of the adjacency matrix via removing edges for three different classes of networks, scale-free, Erdös-Rényi, and geometric networks.

In the first scenario, we consider an Erdös-Rényi graph with 500 nodes and a connection probability of 0.01 . We seek the variation of $\beta_{c}=1 / \lambda_{\max }(\mathbf{A})$ when between 10 and 90 percent of the edges are removed based on the systematic method proposed in this work compared with a random removal procedure where edges are removed based on a uniform distribution. The result is depicted in fig. 1. Clearly, randomly removing edges results in a smaller increase of $\beta_{c}$ (smaller decrease of $\lambda_{\max }(\mathbf{A})$ ) compared to the systematic edge removal procedure proposed in this work.

In the next example we consider a network described by a random geometric graph. Here, a random geometric graph is constructed by randomly and uniformly distributing 100 nodes within a 20 by 20 unit square. Then two nodes are connected if the distance between them is 


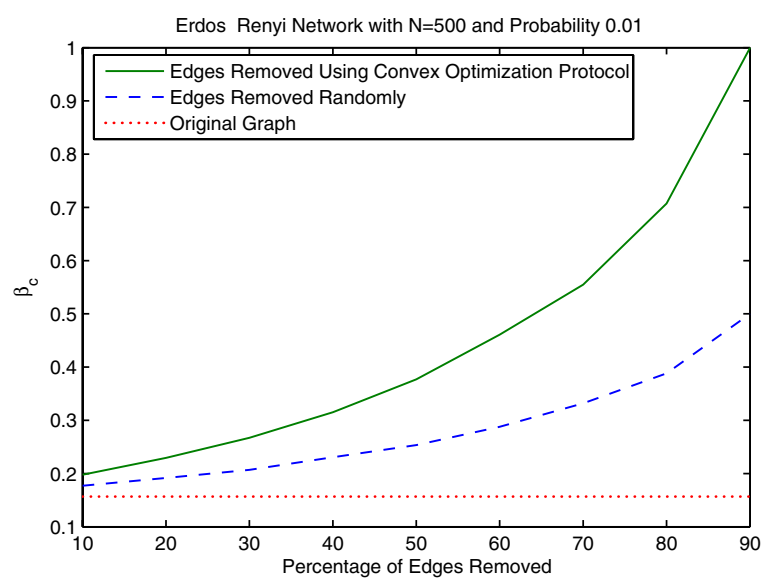

Fig. 1: (Colour online) Increasing the critical probability $\beta_{c}$ in a random Erdös-Rényi network.

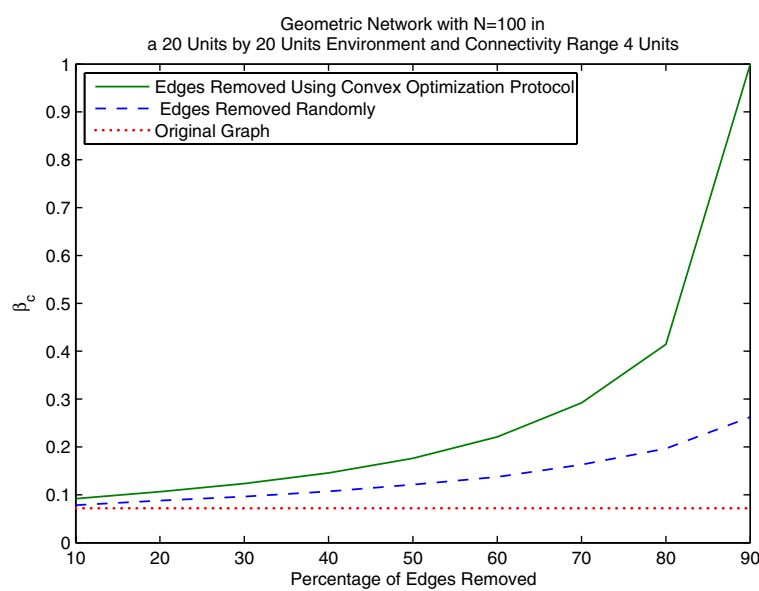

Fig. 2: (Colour online) Increasing the critical probability $\beta_{c}$ in a random geometric graph network.

less than or equal to 4 units. This type of graph is often used as a simplified version of a practical wireless network connectivity model. The variation of $\beta_{c}$ found by removing edges randomly is compared to the edge-removal method proposed in this work. The result is shown in fig. 2 .

Finally, we consider a scale-free network with $N=500$ nodes. Again, we seek the variation of $\beta_{c}=1 / \lambda_{\max }(\mathbf{A})$ and want to compare a scheme where edges are randomly removed according to a uniform distribution with the systematic scheme proposed in this work. The comparison is depicted in fig. 3. The absolute minimum value for $\lambda_{\max }(\mathbf{A})$ is 1 whenever there is at least 1 edge in the graph; i.e. $\lambda_{\max }(\mathbf{A})<1$ implies the graph is edgeless and indeed in an edgeless graph $\lambda_{\max }(\mathbf{A})=0$. After removing approximately $70 \%$ of the edges using the proposed systematic edge-removal scheme we have achieved the absolute minimum value for $\lambda_{\max }(\mathbf{A})$ and therefore maximized $\beta_{c}$ to 1 . Removing additional edges has no additional benefit in this case. This is not true when the edges are removed randomly and one needs to remove significantly larger number of edges. Therefore, as seen in the

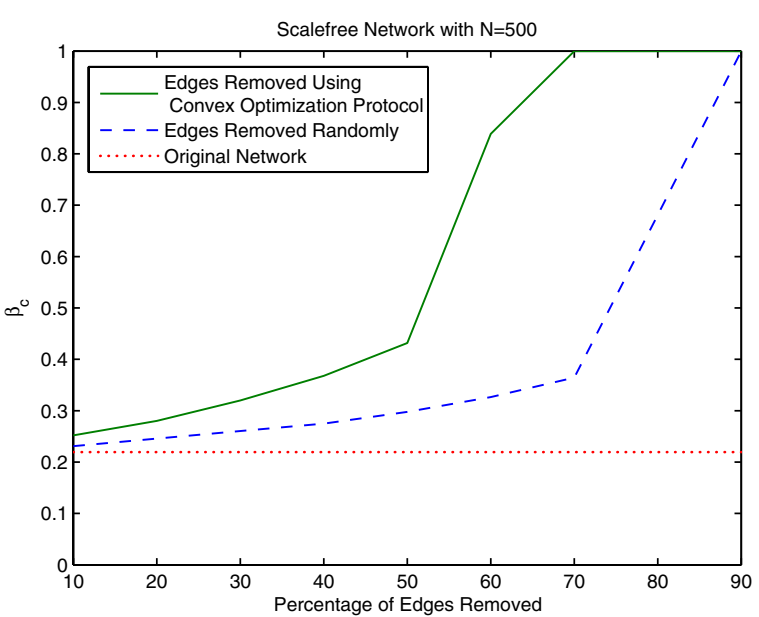

Fig. 3: (Colour online) Increasing the critical probability $\beta_{c}$ in a scale-free network.

figure, the proposed method to remove edges outperforms the a random edge removal scheme significantly.

The idea in each example is to increase the critical probability $\beta_{c}$. A disease is more likely to die out quickly when the infection probability between pairs of nodes $\beta$ is below $\beta_{c}$. Thus, manipulating the network such that $\beta_{c}$ is larger means a greater range of infection probabilities $\beta \in\left[0, \beta_{c}\right)$ will not reduce the ability of the network to autonomously wipe out the disease in a timely fashion.

Concluding remarks. - In this work we considered certain network operations that can be used to slow the spread of diseases in complex network topologies. The approach considered in this work differs from existing techniques in the sense that we optimally remove individual links. Thus, individual nodes are not necessarily immunized in the classical sense but rather we actively control the interactions and the disease's path of movement. We provided a systematic algorithm to achieve this edgewise immunization via a relaxed convex optimization protocol.

This work was supported by the Swedish Research Council, Knut and Alice Wallenberg Foundation and NICTA. NICTA is funded by the Australian Government as represented by the Department of Broadband, Communications and the Digital Economy and the Australian Research Council through the ICT Centre of Excellence program.

\section{REFERENCES}

[1] Barabási A. L., Linked: The New Science of Networks (Perseus Books Group, Cambridge, Mass.) 2002.

[2] Deo N., Graph Theory with Applications to Engineering and Computer Science (PHI Learning Pvt. Ltd.) 2004. 
[3] Scott J., Sociology, 22 (1988) 109.

[4] Newman M. E. J., Watts D. J. and Strogatz S. H., Proc. Natl. Acad. Sci. U.S.A., 99 (2002).

[5] Eubank S., Guclu H., Kumar V. S. A., Marathe M. V., Srinivasan A., Toroczkai Z. and Wang N., Nature, 429 (2004) 180.

[6] Klovdahl A. S., Soc. Sci. Med., 21 (1985) 1203.

[7] Pastor-Satorras R. and Vespignani A., Phys. Rev. Lett., 86 (2001) 3200.

[8] Ganesh A., Massoulié L. and Towsley D. The effect of network topology on the spread of epidemics, in Proceedings of the 24th Annual Joint Conference of the IEEE Computer and Communications Societies (INFOCOM 2005), Vol. 2 (IEEE, 2005) pp. 1455-1466.

[9] Chakrabarti D., Wang Y., Wang C., Leskovec J. and Faloutsos C., ACM Trans. Inf. Syst. Secur., 10 (2008) 1.

[10] Van Mieghem P. and Omic J., In-homogeneous Virus Spread in Networks, Delft University of Technology Report 2008081, 2008.

[11] Van Mieghem P., Omic J. and Kooij R., IEEE/ACM Trans. Netw., 17 (2009) 1.

[12] Gómez S., Arenas A., Borge-Holthoefer J., Meloni S. and Moreno Y., EPL, 89 (2010) 38009.

[13] Pastor-Satorras R. and Vespignani A., Epidemics and immunization in scale-free networks, in Handbook of Graphs and Networks: From the Genome to the Internet (Wiley) 2005, Chapt. 5, pp. 111-130.
[14] Cohen R., Havlin S. and Ben-Avraham D., Phys. Rev. Lett., 91 (2003) 24.

[15] Giakkoupis G., Gionis A., Terzi E. and Tsaparas P., Models and algorithms for network immunization, Technical Report, Department of Computer Science, University of Helsinki, C-2005-75, 2005.

[16] Zou C. C., Towsley D. and Gong W., IEEE Trans. Dependable Secure Comput., 4 (2007) 105.

[17] Meyers L. A., Bull. Am. Math. Soc., 44 (2007) 1.

[18] Madar N., Kalisky T., Cohen R., Ben-avraham D. and Havlin S., Eur. Phys. J. B-Condens. Matter Complex Syst., 38 (2004) 269.

[19] Holme P., Europhys. Lett., 68 (2004) 908.

[20] Miller J. C. and Hyman J. M., Physica A: Stat. Mech. Appl., 386 (2007) 780.

[21] Omic J., Orda A. and Van Mieghem P., Protecting against network infections. A game theoretic perspective, in Proceedings of the 28th Conference on Computer Communications (INFOCOM), Rio de Janeiro, Brazil, 2009, pp. 1485-1493

[22] Boyd S. P. and Vandenberghe L., Convex Optimization (Cambridge University Press) 2004.

[23] Chung F. R. K., Spectral Graph Theory (American Mathematical Society) 1997.

[24] Boyd S., Convex optimization of graph Laplacian eigenvalues, in Proceedings of the 2006 International Congress of Mathematicians, Madrid, Spain, August 2006, pp. 1311-1320. 